\title{
SOCIAL MEDIA ASSESSMENT FOR MARKETING STUDENTS: THE KLOUT CHALLENGE
}

\author{
Todd J. Bacile, Florida State University, U.S.A.
}

\begin{abstract}
This paper discusses a class project created by the author titled: "The Klout Challenge", which helps students gain engagement skills and the capacity to influence within social media. The Klout Challenge helps to fill the void of students failing to develop experience with social media tools for marketing application. This project uses an independent online metric available from Klout.com to assess students' level of social media influence. The impact of this project stretches beyond the classroom, as some hiring firms are beginning to assess applicants' Klout scores. Therefore, students who successfully put effort into this project are likely to appear more attractive as job applicants. Educators can use this paper's five-step implementation plan to adapt this project into a marketing or advertising class.
\end{abstract}

A before and after analysis for one class that participated in this project reveled significantly higher Klout scores after participating. When the project began, most students were below an average Klout score of $20(\mathrm{M}=16.7, \mathrm{SD}=10.7, \mathrm{n}=46)$. Upon project completion Klout scores were significantly higher $(p<.0001, \mathrm{M}=39.1, \mathrm{SD}=11.5, \mathrm{n}=44)$. Students with higher Klout scores said they actively used the social media engagement strategies presented in class. There are two methods an instructor can use for grade assignment. The first grading calculation method is subjective, using the distribution of scores around the mean to assign a letter grade. This method is effective, as it challenges students to compete against one another for a higher score. However, some students prefer fixed grading thresholds known up front. Instructors who prefer to offer fixed grading cutoff points can elect to use a second grading method: take the final Klout score multiplied by 2.00 to arrive at the grade percentage.

References available upon request. 